\title{
The Clinical Features of Asymmetry Weakness in Childhood Guillain- Barre syndrome: The Outcome and 5-year Follow-up
}

Rui-Di Sun

Wuhan Children hosptial https://orcid.org/0000-0002-6607-3195

jun Jiang ( $\nabla_{\text {jiangjuntg@sina.com })}$

Research article

Keywords: Guillain-Barre syndrome, asymmetry weakness, childhood

Posted Date: June 8th, 2020

DOI: https://doi.org/10.21203/rs.3.rs-31710/v1

License: (c) (i) This work is licensed under a Creative Commons Attribution 4.0 International License. Read Full License 


\section{Abstract}

Objectives To compare the clinical profile and long-term outcome of children with asymmetry weakness and symmetry weakness in GuillainBarre syndrome(GBS).

Methods We retrospective analysis the clinical parameters, auxiliary examinations and long-term outcome between asymmetry weakness and symmetry weakness in childhood GBS.

Results A total of 72 children were included, 12 children had asymmetry weakness. Six children were transient asymmetry weakness and six children were persistent asymmetry weakness. Compared to symmetry weakness children, asymmetry weakness had more preschool children (75\% vs $25 \%, P=0.005)$, longer days on hospital(26.5(15-37) days vs 11 (9-15) days, $p=0.000)$, more mechanical ventilation in children(50\% vs $8.33 \%, p=0.000$ ), higher Disease severity score(DSS)at nadir of disease(4(3-5) vs 3(1-4), $p=0.010)$, more axonal subtypes(50\% vs $15 \%$, $p=0.013$ ) and more complications( $58.33 \%$ vs $8.33 \%, p=0.000)$. Eight children had sequelae and sixty-four children had good recovery. Compared to good recovery group, sequelae group had more axonal subtypes(62.5\% vs $15.63 \%, p=0.002)$ and more persistent asymmetry weakness $(62.5 \%$ vs $4.69 \%, p=0.000)$.

Conclusions In conclusion, asymmetry weakness had two types in GBS, namely transient and persistent asymmetry weakness. Asymmetry weakness in GBS indicated more complex condition during disease than symmetry weakness. Persistent asymmetry weakness and axonal subtypes in GBS related with sequelae. Anterior horn cells in the spinal cord involvement may be the possible function in persistent asymmetry weakness combined with axonal subtypes in GBS.

\section{Introduction}

Guillain-Barre syndrome(GBS) is an acute inflammatory polyneuropatchy typically characterized by progressive symmetry weakness and areflexia. GBS is currently the most frequent cause of acute flaccid paralysis in children ${ }^{[1]}$, with an estimated incidence of $0.5: 100$ 000 to 1.5:100 000 individuals in the under 18-year age group ${ }^{[2]}$. It has usually been considered as an immune-mediated polyneuropatchy clinically characterised by acute symmetry muscle weakness and areflexia. Symmetry weakness is the core feature in GBS, but asymmetry weakness was also found in some cases. In pediatric GBS, asymmetry weakness was the atypical clinical feature of GBS and it occured in a significant proportion of children ${ }^{[3]}$. A case report of asymmetry GBS described a patient who developed an acute motor-sensory neuropatchy with a more prolonged and incomplete type of recovery in more weakness limb ${ }^{[4]}$. Until now, the clinical feature of asymmetry GBS was only reported in case reports, and its function was unclear. Indeed, these cases report raised issues on diagnosis and features in asymmetry GBS. The aim of this paper is to describe features in asymmetry GBS, make a comparison between asymmetry weakness and asymmetry weakness in GBS and discuss the possible pathophysiology in this type of GBS.

\section{Materials And Methods}

\section{Children}

We retrospectively reviewed our database of children with a diagnosis of GBS at the neurology department of Wuhan children hospital between 2010 and 2018. The diagnosis was made in each case in accordance with the diagnostic criteria of Asbury and Cornblath ${ }^{5]}$. Recurrent GBS was excluded. We focus on GBS with limbs weakness. The study was approved by the ethical committee of Wuhan Children's Hospital. Written informed consent was obtained from their parents of patient in our study.

\section{Methods}

We systematically collected datas on children's demographics, physical examination, nerve conduction results, CSF, and other related test results. Peak weakness was defined as the nadir of disease. Disease severity score(DSS)was determined by Hughes' functional scale score ${ }^{[6]}$ or upper limb disability grading scale ${ }^{[7]}$ (when the muscle weakness was more prominent in upper limbs). When upper limbs was asymmetry weakness, DSS was evaluated on the more prominent weakness limb. Recovery was defined as a return to normal life with a DSS of 0 . At least one year should be given to follow-up.

All children included had undergone electrophysiological study of at least 4 motor and 2 sensory nerves within 3 weeks from symptom-onset. The electrophysiology abnormalities were categorised as acute inflammatory demyelinating poluradiculoneuropathy (AIDP), and axonal type: acute motor axonal neuropathy(AMAN) acute motor and sensory axonal neuropathy(AMSAN) or unclassifiable (equivocal) based on the single study approach in accordance to Hadden et al.'s and Rajabally et al.'s electrodiagnostic criteria ${ }^{[8]}$. If children had more than two electrophysiological studies, subtype was categorized based on last electrophysiological result. 
Asymmetry weakness was defined as at least 1 score difference by Medical Research Council (MRC) grade between the right- versus left-side muscles in children at admission ${ }^{[9]}$.

\section{Statistical Analysis}

Statistical analyses were performed with SPSS version 11.0 software. Categorical data are presented as proportions, and differences in proportions were tested using the $\chi 2$ test. Continuous data with a normal distribution are presented as the means \pm SDs, and these variables were tested using Student $t$ test or analysis of variance. Continuous variables with a skewed distribution are presented as medians(M) and interquertile range(IQR) ([25th - 75th]), and these variables were tested by the Mann-Whitney $\mathrm{U}$ test. A test level $a=0.05$ or $p<0.05$ indicated that the difference was significant.

\section{Results}

\subsection{Demographics}

In total, we identified 72 children with clinical diagnosis of classic GBS. Among these, 42 were males, 30 were females. 24 children were under 6 -year-old. The median age at time of diagnosis was $91.86 \pm 37.94$ months. Antecedent events were found in 49 of children. 7 children had diarrhoea, 14 children had upper repiratory tract infections(URI), 21 children had fever only, 2 children had vaccination $₫ 2$ children had Chicken pox and 3 children had herpes. The average days at hospital stay was 12(8-18) days. Ten children need MV during the disease. Twelve children had sensory nerve deficit and sixteen had cranial nerve involvement. Signs of dysautonomia were present in 14 children (35\%), 5 of whom had arterial hypertension (58\%), 6 had sinus tachycardia (29\%), 2 had urinary problems (29\%) and 1 had vasomotor disorders ( $8 \%$ ). The mean GBS disability score in nadir was 3(2-4). 18 children had positive ganglioside antibodies in serum and 47 had albuminocytologic dissociation on the cerebrospinal fluid (CSF). 15 children were classified as axonal type of GBS(13 AMAN and 2 AMSAN), 45 were classified as AIDP and 12 were classified as equivocal type. One children had abnormality in cranial MRI. Complications: 12 children had complications, including central nerve system(CNS) infection(abnormal electroencephalogram with limit impaired or normal consciousness), liver function impairment, gastrointestinal bleeding, electrolyte disturbance, cardiac arrest, bronchopneumonia, II respiratory failure, atelectasis, severe protein-energy malnutrition, closed craniocerebral injury. The most common pathogen during disease was mycoplasma pneumonia infection. Intravenous immunoglobulin was the first-line therapy, and some children need plasma exchange or glucocorticoid therapy.

\section{2 clinical feature of asymmetry weakness.}

Twelve children had asymmetry weakness. Nine children were male, three were female. Nine children were under 6-year-old. The median age at time of diagnosis was $51.25 \pm 33.54$ months. Antecedent infections were found in 6 children. The mean length of the hospital stay was $26.5(15-$ 37)days. Six children need MV. One child had sensory nerves deficit, and four children had cranial nerves deficit. The nadir of disease in DSS were 4(3-5). Three children had dysautonomia. Six children had persistently asymmetry weakness and six children had transit asymmetry weakness (Table 1). Among six children with persistently asymmetry weakness, one child had good recovery. The characteristics of the asymmetry weakness children or those asymmetry weakness are shown in Table 1.In six children with transit asymmetry weakness, 5 children(5/6,83.33\%) were AIDP, while in persistent asymmetry weakness, 5 children(5/6, 83.33\%) were axonal GBS, AIDP were more reported in transit asymmetry weakness while axonal GBS were more reported in persistent asymmetry weakness.

Table 1 clinical feature in 12 children with asymmetry weakness $(n=12)$ 


\begin{tabular}{|c|c|c|c|c|c|c|}
\hline & Case 1 & Case 2 & Case 3 & Case 4 & Case 5 & Case 6 \\
\hline Age/sex(years) & $9 / \mathrm{M}$ & $2 / \mathrm{M}$ & $1 / M$ & $7 / M$ & $3 / M$ & $4 / F$ \\
\hline $\begin{array}{l}\text { Antecedent } \\
\text { infections }\end{array}$ & $\mathrm{N}$ & URI & $\mathrm{N}$ & $\mathrm{N}$ & $\mathrm{N}$ & $\mathrm{N}$ \\
\hline Days on hospital & 21 & 16 & 15 & 18 & 13 & 14 \\
\hline Nerve studies & AIDP & AMAN & AIDP & AIDP & AIDP & AIDP \\
\hline $\begin{array}{l}\text { Mechanical } \\
\text { ventilation }\end{array}$ & $\mathrm{N}$ & $\mathrm{N}$ & $\mathrm{N}$ & $P$ & $\mathrm{~N}$ & $\mathrm{~N}$ \\
\hline $\begin{array}{l}\text { Sensory nerves } \\
\text { deficit }\end{array}$ & $\mathrm{N}$ & N & $\mathrm{N}$ & $P$ & $\mathrm{~N}$ & $\mathrm{~N}$ \\
\hline $\begin{array}{l}\text { The muscle } \\
\text { strength at } \\
\text { admission(MRC) }\end{array}$ & $\begin{array}{l}\text { left upper } \\
\text { limb:3/5 } \\
\text { right } \\
\text { upper } \\
\text { limb and } \\
\text { bilateral } \\
\text { limbs:5/5 }\end{array}$ & $\begin{array}{l}\text { left lower } \\
\text { limb:2/5, right } \\
\text { lower } \\
\text { limb: } 4 / 5 \text {,bilateral } \\
\text { upper limbs:5/5 }\end{array}$ & $\begin{array}{l}\text { right upper } \\
\text { limb:2/5,left upper } \\
\text { limb:3/5, bilateral } \\
\text { lower limbs: } 2-15\end{array}$ & $\begin{array}{l}\text { bilateral upper } \\
\text { limbs: } 3^{+} / 5 \text {, left } \\
\text { lower } \operatorname{limb}: 2 / 5 \text {, } \\
\text { right limb } \\
\text { lower:3/5 }\end{array}$ & $\begin{array}{l}\text { right upper } \\
\text { limb:2/5, left upper } \\
\text { limb:4/5,bilateral } \\
\text { lower limbs:2/5. }\end{array}$ & $\begin{array}{l}\text { bilateral upper } \\
\text { limbs:5/5,left } \\
\text { lower limb:3/5, } \\
\text { right limb:4/5 }\end{array}$ \\
\hline $\begin{array}{l}\text { Duration time on } \\
\text { asymmetry } \\
\text { weakness }\end{array}$ & 2 days & 5 days & 4 days & 12 days & 7 days & 4 days \\
\hline $\begin{array}{l}\text { Cranial nerve } \\
\text { deficit }\end{array}$ & $P$ & N & N & $P$ & $\mathrm{~N}$ & $\mathrm{~N}$ \\
\hline Dysautonomia & N & N & $\mathrm{N}$ & N & $\begin{array}{l}\text { hypertension, } \\
\text { hypertensive } \\
\text { encephalopathy. }\end{array}$ & $\mathrm{N}$ \\
\hline $\begin{array}{l}\text { Ganglioside } \\
\text { antibodies }\end{array}$ & N & Anti-GM1 & N & N & N & $\mathrm{N}$ \\
\hline Complications & $\mathrm{N}$ & $\mathrm{N}$ & $\mathrm{N}$ & $\begin{array}{l}\text { gastrointestinal } \\
\text { bleeding }\end{array}$ & $\begin{array}{l}\text { electrolyte } \\
\text { disturbance }\end{array}$ & $\mathrm{N}$ \\
\hline ICU therapy days & $\mathrm{N}$ & $\mathrm{N}$ & $\mathrm{N}$ & 5 days & $\mathrm{N}$ & $\mathrm{N}$ \\
\hline $\begin{array}{l}\text { Cranial and spinal } \\
\text { MRI }\end{array}$ & $\mathrm{N}$ & $\mathrm{N}$ & $\mathrm{N}$ & $\begin{array}{l}\text { Bilateral frontal } \\
\text { white matter: } \\
\text { slightly higer } \\
\text { signal intensity. }\end{array}$ & $\mathrm{N}$ & $\mathrm{N}$ \\
\hline $\begin{array}{l}\text { Pathogen } \\
\text { detection results }\end{array}$ & $\mathrm{N}$ & $\begin{array}{l}\text { mycoplasma } \\
\text { pneumoniae }\end{array}$ & $\mathrm{N}$ & $\mathrm{N}$ & $\begin{array}{l}\text { klebsiella } \\
\text { pneumoniae } \\
\text { enterobacter } \\
\text { cholerae } \\
\text { enterobacter } \\
\text { cloacae }\end{array}$ & $\begin{array}{l}\text { mycoplasma } \\
\text { pneumoniae }\end{array}$ \\
\hline $\begin{array}{l}\text { Albuminocytologic } \\
\text { dissociation on } \\
\text { the CSF }\end{array}$ & $\mathrm{N}$ & $\mathrm{N}$ & $P$ & $\mathrm{~N}$ & Not done & $P$ \\
\hline Therapy & G & IVIG & IVIG & IVIG & IVIG & $\mathrm{N}$ \\
\hline $\begin{array}{l}\text { Recovery } \\
\text { time/follow-up } \\
\text { time }\end{array}$ & 39 days & 41 days & 47 days & 11 months & 8 months & 55 days \\
\hline Sequelae & $\mathrm{N}$ & $\mathrm{N}$ & $\mathrm{N}$ & $\mathrm{N}$ & $\mathrm{N}$ & $\mathrm{N}$ \\
\hline
\end{tabular}

$\mathrm{P}=$ positive, $\mathrm{N}=$ negative, $\mathrm{CNS}=$ central nerve system, The $\mathrm{CSF}=$ cerebrospinal fluid, $\mathrm{MRC}=$ Medical Research Council grade,URI= upper repiratory tract infections, G=glucocorticoid, IVIG= intravenous immunoglobulin, AIDP= acute inflammatory demyelinating poluradiculoneuropathy,AMAN= acute motor axonal neuropathy

,AMSAN= acute motor and sensory axonal neuropathy.

Table 1 clinical feature in 12 children with asymmetry weakness (sequels) $(n=12)$ 


\begin{tabular}{|c|c|c|c|c|c|c|}
\hline & Case 7 & Case8 & Case 9 & Case10 & Case 11 & Case12 \\
\hline Age/sex(years) & $1 / F$ & $4 / \mathrm{M}$ & $5 / \mathrm{M}$ & $7 / F$ & $4 / \mathrm{M}$ & $2 \mathrm{~m}^{\#} / \mathrm{M}$ \\
\hline $\begin{array}{l}\text { Antecedent } \\
\text { infections }\end{array}$ & fever & fever & Fever & $\mathrm{N}$ & Fever,cough & fever \\
\hline Days on hospital & 41 & 37 & 32 & 34 & 84 & 36 \\
\hline Nerve studies & equivocal & AMSAN & AMAN & AMSAN & AMAN & AMAN \\
\hline $\begin{array}{l}\text { Mechanical } \\
\text { ventilation }\end{array}$ & $\mathrm{P}$ & $\mathrm{N}$ & $P$ & $P$ & $P$ & $P$ \\
\hline $\begin{array}{l}\text { Sensory nerves } \\
\text { deficit }\end{array}$ & $\mathrm{N}$ & $\mathrm{N}$ & $\mathrm{N}$ & $\mathrm{N}$ & $\mathrm{N}$ & $\mathrm{N}$ \\
\hline $\begin{array}{l}\text { The muscle } \\
\text { strength at } \\
\text { adimission(MRC) }\end{array}$ & $\begin{array}{l}\text { right upper } \\
\text { limbs: } 3^{+} / 5 \text {,left } \\
\text { upper } \\
\text { limbs: } 2^{+} / 5 \text {,right } \\
\text { lower } \\
\text { limb: } 1 / 5, \text { left } \\
\text { lower limb: } 1^{+} / 5\end{array}$ & $\begin{array}{l}\text { left upper } \\
\text { limb:3/5,right } \\
\text { upper } \\
\text { limb:5/5,bilateral } \\
\text { lower limbs: } 3 / 5\end{array}$ & $\begin{array}{l}\text { left limbs: } 2 / 5 \\
\text { right limbs: } 3^{+} / 5\end{array}$ & $\begin{array}{l}\text { bilateral } \\
\text { upper } \\
\text { limbs: } 1 / 5 \text {, } \\
\text { left lower } \\
\text { limb: } 3^{+} / 5 \text {, } \\
\text { right lower } \\
\text { limb:5/5 }\end{array}$ & $\begin{array}{l}\text { bilateral upper } \\
\text { limbs:2/5, left lower } \\
\text { limbs: } 1 / 5 \text {, right } \\
\text { lower limbs: } 2 / 5 \text {. }\end{array}$ & $\begin{array}{l}\text { right upper } \\
\text { limb:1/5,left upper } \\
\text { limb:0/5, right } \\
\text { lower limb: } 3^{+} / 5 \text {, } \\
\text { left lower limbs:4/5 }\end{array}$ \\
\hline $\begin{array}{l}\text { Duration time on } \\
\text { asymmetry } \\
\text { weakness }\end{array}$ & $\begin{array}{l}\text { persistent } \\
\text { asymmetry } \\
\text { weakness }\end{array}$ & $\begin{array}{l}\text { persistent } \\
\text { asymmetry } \\
\text { weakness }\end{array}$ & $\begin{array}{l}\text { persistent } \\
\text { asymmetry } \\
\text { weakness }\end{array}$ & $\begin{array}{l}\text { persistent } \\
\text { asymmetry } \\
\text { weakness }\end{array}$ & $\begin{array}{l}\text { persistent } \\
\text { asymmetry } \\
\text { weakness }\end{array}$ & $\begin{array}{l}\text { persistent } \\
\text { asymmetry } \\
\text { weakness }\end{array}$ \\
\hline $\begin{array}{l}\text { Cranial nerve } \\
\text { deficit }\end{array}$ & $\mathrm{N}$ & $\mathrm{N}$ & $\mathrm{P}$ & $\mathrm{P}$ & $\mathrm{N}$ & $\mathrm{N}$ \\
\hline Dysautonomia & $\mathrm{N}$ & $\mathrm{N}$ & $\begin{array}{l}\text { Urinary problem, } \\
\text { hypertension }\end{array}$ & $\mathrm{N}$ & $\begin{array}{l}\text { vasomotor } \\
\text { disorders }\end{array}$ & $\mathrm{N}$ \\
\hline $\begin{array}{l}\text { Ganglioside } \\
\text { antibodies }\end{array}$ & $\mathrm{N}$ & $\mathrm{N}$ & Anti-GD1b & $\mathrm{N}$ & Anti-GM1 & Anti-GM1 \\
\hline Complications & $\mathrm{N}$ & CNS infection & CNS infection & $\begin{array}{l}\text { CNS } \\
\text { infection, } \\
\text { Pneumonia }\end{array}$ & $\begin{array}{l}\text { CNS infection, } \\
\text { Cardiac Arrest, } \\
\text { bronchopneumonia, } \\
\text { abnormal liver } \\
\text { function, II } \\
\text { respiratory failure, } \\
\text { atelectasis, severe } \\
\text { protein-energy } \\
\text { malnutrition, closed } \\
\text { craniocerebral } \\
\text { injury }\end{array}$ & $\begin{array}{l}\text { CNS infection, } \\
\text { bronchopneumonia }\end{array}$ \\
\hline ICU therapy days & 12 days & $\mathrm{N}$ & 10 days & 7 days & 42 days & 21 days \\
\hline $\begin{array}{l}\text { Cranial and spinal } \\
\text { MRI }\end{array}$ & $\mathrm{N}$ & $\mathrm{N}$ & $\mathrm{N}$ & $\mathrm{N}$ & $\mathrm{N}$ & $\mathrm{N}$ \\
\hline $\begin{array}{l}\text { Pathogen } \\
\text { detection results }\end{array}$ & $\mathrm{N}$ & $\begin{array}{l}\text { Mycoplasma } \\
\text { pneumoniae }\end{array}$ & Cytomegalovirus & $\mathrm{N}$ & $\mathrm{N}$ & $\mathrm{N}$ \\
\hline $\begin{array}{l}\text { Albuminocytologic } \\
\text { dissociation on } \\
\text { the CSF }\end{array}$ & $P$ & $\mathrm{~N}$ & $\mathrm{~N}$ & $\mathrm{~N}$ & $\mathrm{~N}$ & $\mathrm{~N}$ \\
\hline therapy & $I V I G+G$ & IVIG+PE & IVIG+PE & $I V I G+G$ & $I V I G+P E+G$ & Two circle of IVIG \\
\hline $\begin{array}{l}\text { Recovery } \\
\text { time/follow-up } \\
\text { time }\end{array}$ & 4 years & 19 months & 37 months & 28 months & 16 months & 5 years \\
\hline Sequelae & $\begin{array}{l}\text { Right upper } \\
\text { limbs:5/5,left } \\
\text { upper } \\
\text { limbs:3+/5,right } \\
\text { lower } \\
\text { limb:3/5,left } \\
\text { lower limb:4/5 }\end{array}$ & $\mathrm{N}$ & $\begin{array}{l}\text { Left limbs:3/5, } \\
\text { right limbs:5/5 }\end{array}$ & $\begin{array}{l}\text { Bilateral } \\
\text { upper } \\
\text { limbs: } 3 / 5 \text {, } \\
\text { bilateral } \\
\text { lower } \\
\text { limb:5/5 }\end{array}$ & $\begin{array}{l}\text { Bilateral upper } \\
\text { limbs:3/5, bilateral } \\
\text { lower limbs: } 4 / 5 \text {. }\end{array}$ & $\begin{array}{l}\text { Right upper } \\
\text { limb:4/5,left upper } \\
\text { limb:2/5, right } \\
\text { lower limb:4+/5, } \\
\text { left lower limbs:5/5 }\end{array}$ \\
\hline
\end{tabular}


$\mathrm{P}=$ positive, $\mathrm{N}=$ negative, $\mathrm{CNS}=$ central nerve system, The $\mathrm{CSF}=$ cerebrospinal fluid, $2 \mathrm{~m}^{\#}=2$ months, $\mathrm{MRC}=$ Medical Research Council grade, $\mathrm{PE}=$ plasma exchange, $\mathrm{G}=$ glucocorticoid, $\mathrm{IVIG}=$ intravenous immunoglobulin. $\mathrm{MRC}=$ Medical Research Council grade. $\mathrm{AIDP}=$ acute inflammatory demyelinating poluradiculoneuropathy,AMAN= acute motor axonal neuropathy,AMSAN= acute motor and sensory axonal neuropathy.

\section{Table 1 clinical feature in 12 children with asymmetry weakness $(n=12)$}

\subsection{Comparative between children with asymmetry weakness and symmetry weakness}

12 children(12/72,16.67\%) had asymmetry weakness. Compared to symmetry weakness group, asymmetry weakness had more preschool children, longer days on hospital, more MV, higher DSS, more axonal type, more complications and sequelae $(\mathrm{P}<0.05)($ Table 2$)$.

Table 2 clinical feature of asymmetrical weakness and symmetrical weakness $(n=72)$

\begin{tabular}{|c|c|c|c|c|}
\hline & Asymmetrical weakness $(n=12)$ & symmetrical weakness $(n=60)$ & $T / x^{2}$ & $P$ \\
\hline Median age(month) & $51.25 \pm 33.54$ & $99.98 \pm 33.46$ & 4.604 & 0.000 \\
\hline Male:female & $9: 3$ & $36: 24$ & 0.947 & 0.331 \\
\hline Preschool children & $9(75 \%)$ & $15(25.00 \%)$ & 7.887 & 0.005 \\
\hline Antecedent events & $6(50.00 \%)$ & $43(71.65 \%)$ & 2.129 & 0.145 \\
\hline Days on hospital & $26.5(15-37)$ & $11(9-15)$ & 4.393 & 0.000 \\
\hline Mechanical ventilation & $6(50.00 \%)$ & $4(8.33 \%)$ & -6.079 & 0.000 \\
\hline Sensory nerves deficit & $1(1.67 \%)$ & $11(18.33 \%)$ & 0.710 & 0.399 \\
\hline Cranial nerves deficit & $4(33.33 \%)$ & $12 \searrow 20 \% \bigotimes$ & 1.014 & 0.314 \\
\hline Dysautonomia & $3(25.00 \%)$ & $11 \rrbracket 18.33 \% \bigotimes$ & 0.280 & 0.597 \\
\hline DSS & $4(3-5)$ & $3(1-4)$ & 2.565 & 0.010 \\
\hline AIDP & $5 \rrbracket 41.67 \% \rrbracket$ & $40(66.67 \%)$ & & \\
\hline Axonal type(AMAN,AMSAN) & $6(50.00 \%)$ & $9(15.00 \%)$ & 6.166 & 0.013 \\
\hline Equivocal type & $1(8.33 \%)$ & $11(18.33 \%)$ & 0.710 & 0.399 \\
\hline ganglioside antibody & $4(33.33 \%)$ & $14(23.33 \%)$ & 0.526 & 0.468 \\
\hline Complication & $7(58.33 \%)$ & $5(8.33 \%)$ & 17.750 & 0.000 \\
\hline \multicolumn{5}{|l|}{ therapy } \\
\hline Monotherapy & $7(58.33 \%)$ & $44(73.33 \%)$ & & \\
\hline Combination therapy & $5 \rrbracket 41.67 \% \rrbracket$ & $6(10.00 \%)$ & 5.742 & 0.017 \\
\hline Sequelae & $5 \rrbracket 41.67 \% \rrbracket$ & $3(5.00 \%)$ & 13.423 & 0.000 \\
\hline
\end{tabular}

AIDP= acute inflammatory demyelinating poluradiculoneuropathy, DSS=Disease severity score.

\section{Table 2 clinical feature of asymmetry weakness and asymmetry weakness $(n=72)$}

3.4 Comparative between children with sequelae and good prognosis group

Eight children had sequelae and sixty-four children had good prognosis. Compared to good prognosis group, sequelae group had younger age at the onset of disease, longer days on hospital, more MV, more axonal subtype, more persistent asymmetry weakness and complications $(\mathrm{P}<0.05)($ Table 3$)$.

Table 3 clinical feature between children with sequelaelae and good prognosis group $(n=72)$ 


\begin{tabular}{|c|c|c|c|c|}
\hline & Sequelae cases $(n=8)$ & recovery cases $(n=64)$ & $T / x^{2}$ & $\mathrm{P}$ \\
\hline Median age(month) & $67.25 \pm 35.10$ & $94.94 \pm 37.40$ & 1.986 & 0.051 \\
\hline Male:female & $5: 3$ & $37: 27$ & 0.063 & 0.801 \\
\hline Preschool children & $5 \llbracket 62.5 \% \rrbracket$ & $19 \llbracket 29.69 \% \rrbracket$ & 3.397 & 0.065 \\
\hline Antecedent events & $5 \llbracket 62.5 \% \rrbracket$ & $44 \llbracket 68.75 \% \rrbracket$ & 0.126 & 0.723 \\
\hline Days on hospital & $33(15-41)$ & $12(9-15)$ & 3.572 & 0.000 \\
\hline Mechanical ventilation & $6 \otimes 75 \% \bigotimes$ & $4(6.25 \%)$ & 28.623 & 0.000 \\
\hline Cranial nerves deficit & $4(50.00 \%)$ & $12(18.75 \%)$ & 3.962 & 0.047 \\
\hline Dysautonomia & $3(37.5 \%)$ & $11(17.19 \%)$ & 1.847 & 0.174 \\
\hline Axonal type & $5 \rrbracket 62.5 \% \rrbracket$ & $10 \rrbracket 15.63 \% \rrbracket$ & 9.432 & 0.002 \\
\hline Persist asymmetrical weakness & $5 \llbracket 62.5 \% \rrbracket$ & $3(4.69 \%)$ & 23.730 & 0.000 \\
\hline Complications & $4(50.00)$ & $8(12.50 \%)$ & 7.100 & 0.008 \\
\hline
\end{tabular}

Table 3 clinical feature between children with sequelae and good prognosis group $(n=72)$

\section{Discussion}

In our study, 16.67\%(12/72) children had asymmetry weakness on admission and this data was lower than previous report which included chronic inflammatory demyelinating polyradiculoneuropatchy ${ }^{[3]}$. GBS is a generalised but unevenly distributed illness ${ }^{[10]}$. In six cases, asymmetry weakness reappeared within 4 weeks, indicating transient asymmetry weakness at admission. In early stage of disease, demyelination was patchy in motor nerves which indicated normal to various abnormalities in electrophysiology. Also, demyelination parameters and motor nerves involvement were in varying degrees. Distal motor latency and conduction block were the most common abnormal parameters in AIDP and median nerve was the most frequently affected nerve ${ }^{[11]}$. In axonal GBS, reduced motor amplitudes was the most common parameter, and the most frequently abnormal nerve was tibial nerve ${ }^{[11]}$. In another investigation, ulnar, peroneal nerve abnormal and sural-sparing pattern were detected at very early stage ${ }^{[12]}$. Several early and sensitive parameters in AIDP, such as medial plantar sensory response or erb's point stimulation method, can be used in the early stage of GBS, as parasthesias often involved the medial plantar nerve or proximal nerve was the first involvement place ${ }^{[13,14]}$. The neurophysiological and pathological findings of AIDP indicated that demyelination of motor nerves is focal and patchy and most of transit asymmetry weakness were AIDP. Persistent asymmetry is one of the features casting doubt or excluding other possible diagnosis. As no reliable serological marker of GBS is currently available, long-time follow-up is necessary to exclude other possible diagnosis. In our asymmetry weakness, at least one year follow-up time was given to exclude other diagnosis.

We also compare clinical features and auxiliary examinations between asymmetry weakness and asymmetry weakness in GBS. Preschool children had more asymmetry weakness than school-age children. The diagnosis of GBS should be carefully evaluated in preschool children as more atypical clinical presentations were reported ${ }^{[15]}$. MV, axonal type as well as combination therapy were more reported in asymmetry weakness. Longer days on hospital, higher DSS in motor function, more complications and sequelae were also reported in asymmetry weakness. Our datas indicated that asymmetry weakness had more serious and complex conditions during disease. Monotherapy had limited effect and combination therapy was necessary to improve prognosis. We also made a comparative between GBS with or without sequelae group which indicated persistent asymmetry weakness was related to sequelae.

Also, 5 patients(5/6,83.33\%) with persistent asymmetry weakness were axonal type. There were several possible interpretations of persistent asymmetry weakness and sequelae in axonal type. First of all, virus infection could cause direct or indirect damage in anterior horn cells in the spinal cord which was the most common asymmetry weakness in neuromuscular disease ${ }^{[16]}$. There were several common features between anterior horn cells in the spinal cord involvement and persistent asymmetry weakness in our axonal cases: central nerves infectious, persistent asymmetry weakness, reduced compound muscle amplitude potentials in motor nerve conduction, and long-time or incomplete recovery. In our hypothesis, there was a virus infection which can bring involvement of peripheral nerves and anterior horn cells in the spinal cord, causing axonal type in nerve conduction, persistent asymmetry weakness and long-time or incomplete recovery. In addition, it should be recognized that axonal GBS and involvement of anterior horn cells in the spinal cord were hardly be distinguished from each other sometimes. In our cases, axonal GBS had positive ganglioside antibodies or involvement of sensory nerves which favor GBS diagnosis. Secondly, there were increased inflammatory cell infiltrates comprising lymphocytes and macrophages in the spinal cord of GBS ${ }^{[17]}$. Impairment of the spinal anterior horn cells with their axons was suggested to be responsible for prolonged motor symptoms, persistent asymmetry weakness and the predominantly axonal type at least as a late-stage feature with poor outcome ${ }^{[17]}$.

Page $7 / 9$ 
As we all known, with deeper and further investigations, many atypical features and variants in GBS were reported. More and more investigations had found that central nerve and peripheral nerves can both involved in GBS, such as encephalopatchy, seizure concurrent with GBS ${ }^{[18,19]}$. Also, peripheral nerves involvement could progress to anterior horn cells in spinal cord in GBS, either by inflammatory cells infiltrated or virus infection.

In conclusion, asymmetry weakness had two types in GBS, including transient and persistent asymmetry weakness. Asymmetry weakness in GBS indicated more complex condition during disease. Persistent asymmetry weakness in GBS had related with sequelae. Anterior horn cells in the spinal cord involvement may be the possible function in persistent asymmetry weakness in GBS.

\section{Abbreviations}

GBS= Guillain-Barré syndrome

DSS=Disease severity score

AIDP= acute inflammatory demyelinating poluradiculoneuropathy

AMAN= acute motor axonal neuropathy

AMSAN= acute motor and sensory axonal neuropathy

MRC=Medical Research Council

URI= upper repiratory tract infections

CSF= cerebrospinal fluid

CNS $=$ central nerve system

\section{Declarations}

Ethics approval and consent to participate

We confirm that we have read the Journal's position on issues involved in ethical publication and affirm that this report is consistent with those guidelines. We had ethics approval and consent to participate.

The study was approved by the ethical committee of Wuhan Children's Hospital. Written informed consent was obtained from their parents of patient in our study.

\section{Competing interests}

None of the authors have any conflicts of interest to disclose.

\section{Availability of data and material}

The datas in report had availability of data and material.

\section{Funding}

There is no funding in this report.

\section{Authors' contributions}

Sun Rui-di designed the study, performed the research, and wrote the paper.

Jiang Jun analysed data,

\section{Acknowledgements}

Thanks to Wuhan Children's Hospital in favor of research.

\section{Consent for Publication}


Images and clinical details of patient were all in compromise anonymity and they consent for the publication of identifying that. A statement of consent was abtained from the patients' guardians to agree to use images or other personal/clinical details.

\section{Reference}

[1] Yuki N, Hartung HP. Guillain-Barre 'syndrome. N Engl J Med 2012;366:2294-2304.

[2]. Rabie M, Nevo Y. Childhood acute and chronic immunemediated polyradiculoneuropathies..

Eur J Paediatr Neurol 2009;13:209-218

[3] Yosha-Orpaz N, Aharoni S, Rabie M, Nevo Y. Atypical Clinical Presentations of Pediatric Acute Immune-Mediated Polyneuropatchy. J Child Neurol 2019;34:268-276.

[4] Logullo F, Manicone M, Di Bella P, Provinciali L. Asymmetry Guillain-Barré syndrome. Neurol Sci . 2006;27:355-359.

[5] Asbury AK, Cornblath DR. Assessment of Current Diagnostic Criteria for Guillain-Barre Syndrome. Ann Neurol 1990;27:S21-24.

[6] Greenwood RJ, Newsom-Davis J, Hughes RA, Aslan S, Bowden AN, Chadwick DW et al. Controlled trial prednisolone in acute polyneuropatchy. Lancet 1978:750-753.

[7] Samadi M, Kazemi B, Golzari Oskoui S, Barzegar M. Assessment of Autonomic Dysfunction in Childhood Guillain-Barré Syndrome. J Cardiovasc Thorac Res 2013;5:81-85.

[8] Rajabally YA, Durand MC, Mitchell J, Orlikowski D, Nicolas G. Electrophysiological diagnosis of Guillain-Barré syndrome subtype: could a single study suffice?. J Neurol Neurosurg Psychiatry. 2015;86:115-9.

[9] Roodbol J, de Wit MY, van den Berg B, Kahlmann V, Drenthen J, Catsman-Berrevoets CE, et al. Diagnosis of Guillain-Barre ' syndrome in children and validation of the Brighton criteria. J Neurol 2017;264:856-861.

[10] Brown WF, Snow R. Patterns and severity of conduction abnormalities in Guillain-Barré syndrome. J Neurol Neurosurgery Psychiatry 1991;54:768-774.

[11] Rajabally YA, Hiew FL. Optimizing electrodiagnosis for Guillain-Barré syndrome: clues from clinical practice. Muscle Nerve 2017;55:748751.

[12] Jin J, Hu F, Qin X, Liu X, Li M, Dang Y, et al. Very Early Neurophysiological Study in Guillain-Barre Syndrome. Eur Neurol 2018;80:100-105.

[13] Ahdab R, Ayache S, Noureldine MHA, Nordine T, Lefaucheur JP. The medial plantar sensory response:A sensitive marker of acute inflammatory demyelinating polyneuropatchy. Clin Neurophysiol. $2017 ; 128: 2122-2124$.

[14] Ye Y, Zhu D, Liu L, Wang K, Huang K, Hou C. Electrophysiological measurement at Erb's point during the early stage of Guillain-Barre syndrome.J Clin Neurosci 2014;21: 786-789.

[15] Roodbol J , de Wit MC, Walgaard C, de Hoog M, Catsman-Berrevoets CE, Jacobs BC. Recognizing Guillain-Barré syndrome in preschool children. Neurology 2011;76:807-10.

[16] Leis AA, Stokic DS .Neuromuscular manifestation of West Nile virus infection. Front Neurol. 2012;21:37.

[17] Müller HD , Beckmann A, Schröder JM. Inflammatory infiltrates in the spinal cord of children with Guillain-Barré syndrome. Acta Neuropathol 2003;106:509-17.

[18] Panda PK, Sharawat IK. Seizure in a child with Guillain-Barre syndrome:Association or coincidence. Indian Pediatr 2020; $57(1): 79$.

[19] Rigamonti A, Basso F, Scaccabarozzi C, Lauria G. Posterior reversible encephalopatchy as the initial manifestation of a Guillain-Barre syndrome. Neuromuscul Disord 2009;19:709-71 